PAPER

\title{
Donepezil for dementia in Parkinson's disease: a randomised, double blind, placebo controlled, crossover study
}

\author{
B Ravina, M Putt, A Siderowf, J T Farrar, M Gillespie, A Crawley, H H Fernandez, \\ M M Trieschmann, S Reichwein, T Simuni
}

See Editorial Commentary, p 903

J Neurol Neurosurg Psychiatry 2005;76:934-939. doi: 10.1136/jnnp.2004.050682

See end of article for authors' affiliations

Correspondence to:

Correspondence to:
Dr B Ravina, Program Director, Clinical Trials, NINDS, Neuroscience Center Rm 2225, 6001 Executive Blvd, Rockville, MD 20892-9257 USA; ravinab@ninds.nih.gov

Received 26 July 2004

In revised form

9 December 2004

Accepted

17 December 2004

\begin{abstract}
Objective: To study the safety and efficacy of a cholinesterase inhibitor, donepezil hydrochloride, for the treatment of dementia in Parkinson's disease (PD).

Methods: This was a randomised double blind, placebo controlled, crossover study in 22 subjects with PD and dementia. Participants were randomised to receive either donepezil followed by identical placebo, or placebo followed by donepezil. Donepezil was administered at 5-10 mg/day. Treatment periods were 10 weeks with a washout period of 6 weeks between the two periods. The primary outcome measure was the Alzheimer's Disease Assessment Scale Cognitive Subscale (ADAScog).

Results: Donepezil was well tolerated and most adverse events were mild. There was no worsening of PD symptoms as measured by the total or motor sections of the Unified Parkinson's Disease Rating Scale.

There was a 1.9 point trend toward better scores on the ADAScog on treatment compared with placebo that was not statistically significant. The secondary cognitive measures showed a statistically significant 2 point benefit on the Mini Mental Status Examination and no change on the Mattis Dementia Rating Scale (MDRS). The Clinical Global Impression of Change (CGI) showed a significant 0.37 point improvement on donepezil. No improvement was observed on the MDRS or the Brief Psychiatric Rating Scale. Carryover between treatment periods was observed but was not statistically significant.

Conclusions: Donepezil was well tolerated and did not worsen PD. There may be a modest benefit on aspects of cognitive function. The possible clinical benefit measured by CGI was reflected in only one of the cognitive scales used in this study.
\end{abstract}

loss in the nucleus basalis of Meynert, ${ }^{12}{ }^{13}$ and this neuropathological finding is associated with the degree of dementia. ${ }^{10}{ }^{13}$ Similarly, levels of choline acetyltransferase from post-mortem samples have been correlated with the degree of dementia in PD. ${ }^{10}$

Donepezil (donepezil hydrochloride) is a well tolerated acetylcholinesterase inhibitor that has been shown to improve cognitive function and activities of daily living in AD. ${ }^{14}{ }^{15}$ Donepezil reduces the hydrolysis or degradation of acetylcholine $^{16}$ and may improve cognitive function in PD through this mechanism. However, anticholinergic agents are sometimes used to treat $\mathrm{PD}$, and cholinergic agents have been reported to worsen motor aspects of $\mathrm{PD} .{ }^{17}$ Thus, the net effect or benefit of cholinergic therapy for PD is uncertain and warrants testing in a clinical trial. Two small, single centre clinical trials in PD patients with dementia have shown mixed results, with one study showing modest but statistically significant changes in the Mini Mental Status Examination (MMSE) and the other showing no change in the MMSE or other global cognitive scales. ${ }^{18}{ }^{19}$ Here we report the results of a double blind, randomised, placebo controlled, crossover trial of donepezil in 22 subjects with PD and dementia.

Abbreviations: AD, Alzheimer's disease; ADAScog, Alzheimer's Disease Assessment Scale Cognitive Subscale; BPRS, Brief Psychiatric Rating Scale; CGI, Clinical Global Impression of Change; CIBIC,

Clinicians' Interview Based Impression of Change; DLB, dementia with Lewy bodies; DSM IV, Diagnostic and statistical manual of mental disorders, 4th ed; MDRS, Mattis Dementia Rating Scale; MMSE, Mini Mental Status Examination; PD, Parkinson's disease; UPDRS, Unified Parkinson's Disease Rating Scale the reduction of cholinergic activity in vivo. ${ }^{11}$ Reduction of cholinergic activity in PD is thought to be secondary to cell

wh. 


\section{METHODS}

\section{Organisation}

The National Institute of Neurological Disorders and Stroke (NINDS) Clinical Center was the coordinating centre. There was an independent medical monitor from the University of Pennsylvania. Donepezil was purchased from Pfizer. Identical placebos were prepared by the University of Pennsylvania Investigational Drug Services Unit, which also bottled and distributed study drug. The study was funded by the NINDS and the University of Pennsylvania.

\section{Recruitment and randomisation}

Recruitment of subjects was performed at the NINDS, Brown University, The University of Pennsylvania, and Northwestern University after each site obtained institutional review board approval of the protocol and an informed consent document from each subject. Inclusion criteria for PD required that the subject be over 40 years of age and have a clinical diagnosis of idiopathic PD defined as at least two of three cardinal features of parkinsonism, with at least one being tremor or rigidity, and a significant and sustained response to dopaminergic medications. ${ }^{2}$ All diagnoses were made by movement disorders specialists. Mild to moderately demented patients were included if they fulfilled the Diagnostic and statistical manual of mental disorders, 4th ed (DSM IV) criteria for dementia and had a MMSE between 17 and 26, inclusive. In order to fulfil DSM IV criteria, the subject must have had impairment in memory and one other cognitive domain, usually executive dysfunction, causing impairment in occupational or social functioning. This was determined clinically, from the history provided by the participant and caregiver, and neurological examination. The dementia had to have developed at least 12 months after the motor manifestations of PD to exclude patients with clinically diagnosed DLB. ${ }^{7}$ Exclusion criteria were $(a)$ alternative sources of dementia such as stroke or metabolic disturbances; (b) pregnancy or lactation; (c) the use of cholinergic or anticholinergic agents except amantadine or tolterodine within the 2 weeks prior to screening; or (d) medical conditions or uncontrolled psychosis that would, in the judgement of the investigator, interfere with the safe conduct of the study.

\section{Study design}

Fig 1 shows a schema of the study design. At the screening visit, the nature, purpose, risks, and requirements of the study were explained to potential participants. After discussing and signing the informed consent document, participants had a complete history, physical and neurological examinations, and MMSE to confirm the diagnosis of PD and dementia. Blood was drawn for vitamin B12 levels, thyroid function tests, and rapid plasma reagen test to exclude other causes of dementia. Pre-menopausal subjects had a urine pregnancy test. The baseline visit occurred within 1 week of the screening visit and included all efficacy assessments. Subjects were then randomised and study drug for the first period was dispensed at the end of the baseline visit.

In the first period (from baseline to week 10) of this crossover study, an investigator contacted the participant by phone at week 4 to ask about side effects and tolerability. At that time, the study drug was increased from $5 \mathrm{mg} /$ day to $10 \mathrm{mg} /$ day or matching placebo. If the higher dose was not tolerated, the lower dose was continued. Safety and efficacy evaluations were performed at weeks 7 and 10. An open label washout occurred from weeks 10-16, during which the subjects did not take any study drug. Study procedures were identical in the second period (weeks 16-26), with a baseline evaluation at week 16 followed by phone contact and

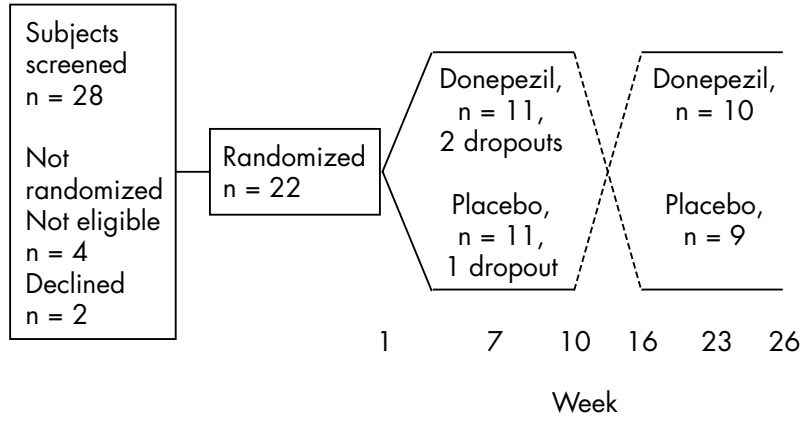

Figure 1 Study schema.

titration as tolerated at week 20, and study visits at weeks 23 and 26.

\section{Intervention and randomisation}

Study drug was dispensed as $5 \mathrm{mg}$ capsules or matching placebo, to be taken orally. Drug was taken once per day for the $5 \mathrm{mg}$ dose and twice a day for the $10 \mathrm{mg}$ dose. Kits were supplied for period 1 and then period 2 at the respective baseline visits. Drug distribution to the sites and randomisation were performed by the University of Pennsylvania Investigational Drug Services Unit. Subjects were randomised in blocks of four to receive either donepezil in period I and placebo in period II or placebo in period I and donepezil in period II.

\section{Outcomes}

The primary outcome measure was the Alzheimer's disease Assessment Scale Cognitive Subscale (ADAScog). ${ }^{20}{ }^{21}$ The MMSE and Mattis Dementia Rating Scale (MDRS)22 were secondary cognitive scales. Other efficacy measures included the Clinical Global Impression of Change (CGI), and a measure of psychosis, the Brief Psychiatric Rating Scale (BPRS) ${ }^{23}$ Safety was assessed by questioning subjects at each phone contact and visit for side effects and intercurrent medical problems. The impact on motor function and parkinsonism was measured by the Unified Parkinson's Disease Rating Scale (UPDRS). ${ }^{24}$

Table 1 Baseline scores and medication use

\begin{tabular}{lll}
\hline Characteristic & $\begin{array}{l}\text { Donepezil/ } \\
\text { placebo } \\
(\mathbf{n}=9)\end{array}$ & $\begin{array}{l}\text { Placebo/ } \\
\text { donepezil } \\
(\mathbf{n}=10)\end{array}$ \\
\hline Age & $75.0(9.8)$ & $72.1(8.1)$ \\
M/F & $9 / 0$ & $6 / 4$ \\
ADAScog total & $29.5(13.5)$ & $32.3(9.5)$ \\
MMSE & $23.1(2.5)$ & $21.4(3.4)$ \\
Total UPDRS & $64.9(25.9)$ & $65.8(21.7)$ \\
Motor UPDRS & $41.2(17.4)$ & $41.2(17.6)$ \\
Duration of PD & $7.1(2.6)$ & $14.4(13.1)$ \\
Use of levodopa (+/- COMT & 10 & 9 \\
inhibitor) & & 3 \\
Use of dopamine agonsits & 3 & 0 \\
Use of selegiline & 0 & 3 \\
Use of neuroleptic & 4 & \\
(quetiapine) & & \\
(10) & &
\end{tabular}

Values are mean (SD). ADAScog, Alzheimer's disease rating scale, cognitive scale (higher scores indicate more impairment); MDRS, Mattis Dementia Rating Scale (lower scores worse); MMSE, Mini Mental Status (lower scores worse); UPDRS, Unified Parkinson's Disease Rating Scale (higher scores worse). COMT, catechol-O-methyltransferase. 


\section{Statistical considerations}

The study was designed to have $\geqslant 80 \%$ power to detect a 4 point or greater difference between donepezil and placebo on the ADAScog, requiring a sample size of 22 subjects. Differences in the categorical variables between groups were assessed using $\chi^{2}$ or Fisher's exact tests. To estimate the treatment effect, the results of the two measurements for each period (weeks 7 and 10 or weeks 23 and 26) were averaged for each subject, and the difference between the means on the two periods used as the combined outcome for each subject. We then formed the mean treatment effect for each sequence and compared the means of the two sequences using a two sample $t$ test for normally distributed variables. ${ }^{25}$ For non-normally distributed variables, a Wilcoxon rank sum test was used to test for differences in the median responses on the two treatments. The Hommel method, a modified Bonferroni correction, was used to adjust the $\mathrm{p}$ values for multiple testing associated with the five outcomes. ${ }^{26}$ To test for carryover, the difference between weeks 16 and 1 was constructed, and a two sample $t$ test (Wilcoxon rank sum test) was used to determine whether there were differences between the means (medians) in the donepezil/placebo group versus the placebo/donepezil group. For the primary outcome, a secondary analysis was carried out by fitting a repeated measures model that adjusted for both period and sequence effects. Additionally, univariate regression analyses were performed to assess the relationship between baseline variables and the observed treatment effect on the primary outcome. Missing data were imputed using the last observation carried forward. No imputation across periods was performed, and participants had to have at least one visit in the second period to be included in the efficacy analyses. Data were analysed using STATA (version 7; College Station, TX, USA) and R (version 2.0; Vienna, Austria) software.

\section{RESULTS}

There were 28 patients were screened, of whom 22 were randomised to the order of administration from October 2001 to February 2003 (fig 1). At baseline, the two groups were similar in age, UPDRS scores, and degree of cognitive impairment on all three cognitive scales (table 1). Subjects in the donepezil/placebo group had a shorter duration of PD: 7.1 (2.6) years compared with 14.4 (13.1) years on average $(p=0.09)$. Three subjects withdrew from the study during the first period. These subjects were included in the safety analysis but not in the efficacy analysis $(n=19)$, because the crossover requires data from both periods to be used in a

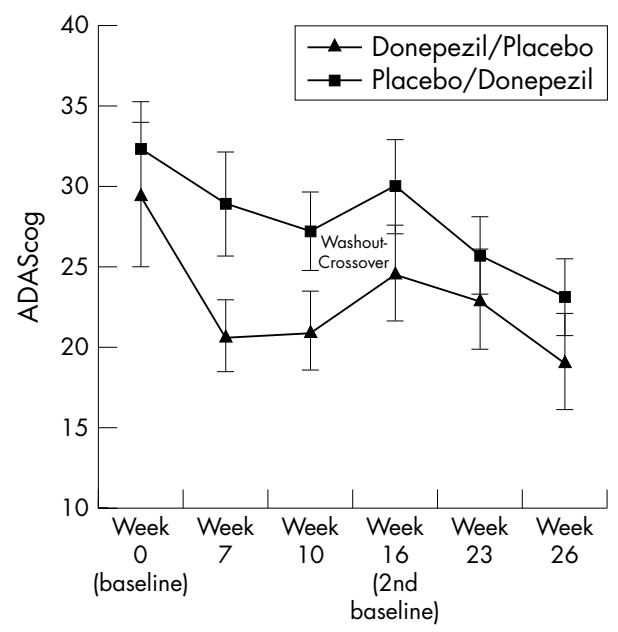

Figure 2 Change in ADAScog scores for both treatment sequences. paired analysis. In the second period, two subjects discontinued use of donepezil and one discontinued use of placebo, but remained in the trial. These subjects were included in efficacy analyses.

\section{Efficacy analyses}

The primary analysis showed a non-significant improvement on the ADAScog on drug compared with placebo (fig 2, table 2). Average (SE) scores on the ADAScog on donepezil were 1.9 (1.4) points better (lower) than average scores on placebo $(p=0.18)$. MMSE scores were $2.0(0.61)$ points better (higher) on donepezil compared with placebo and this reached statistical significance $(p=0.0044)$. There was no change on the MDRS or on the MDRS subsections of attention, initiation/perseveration. The CGI showed significant improvement in favour of donepezil $(p=0.0056)$ and there was no difference between treatment periods on the BPRS (table 3). The observed benefits were not significantly associated with age, sex, or duration of PD in univariate analysis. Carryover effects in the same direction as treatment effects were observed on the cognitive measures, but these effects were not statistically significant (table 4). A repeated measures model that considered each point individually instead of averaging across weeks, and that, adjusted for both period and sequence effects on the ADAScog, approached statistical significance with a $1.9(0.97)$ point improvement in donepezil compared with placebo $(\mathrm{p}=0.055)$.

\section{Safety and tolerability analyses}

The study drug was well tolerated and did not exacerbate PD symptoms (table 5). In the first period, three subjects withdrew from the study: two on donepezil (worsening psychosis and worsening arrhythmia) and one on placebo (worsening psychosis). In the second period, there were no withdrawals, but two subjects discontinued use of donepezil and one subject discontinued use of placebo. Overall tolerability, defined as the proportion of subjects remaining on study drug for the full period, was not significantly different between donepezil and placebo $(p=0.41)$. Adverse effects were experienced by $11 / 21(52 \%)$ subjects while on donepezil and 9/20 (45\%) subjects on placebo. Worsening psychosis and agitation were the most common adverse effects, and occurred with nearly equal frequency in the two groups. In one subject, worsening visual hallucinations led to discontinuation of study drug in the second period while on placebo. There was no evident impact of treatment on total UPDRS or on the motor section of the UPDRS.

\section{DISCUSSION}

In this study, donepezil had a modest effect on cognitive function, primarily evident on the MMSE, and on overall clinical impression, as measured by the CGI. The primary outcome measure of this trial, the ADAScog, showed a trend in favour of donepezil. There was no apparent benefit on the MDRS. The effects on the MMSE and CGI remained statistically significant after adjustment for the five efficacy analyses. These results are consistent with those of Aarsland et al, which showed a statistically significant 1.8 point MMSE improvement on donepezil and a significant improvement on the Clinicians' Interview Based Impression of Change (CIBIC). ${ }^{18}$ The improvement in CGI seen in this trial and on the CIBIC in the Aarsland study argue for a small, but clinically notable benefit that may not be fully captured by cognitive scales.

The differences in results between the three cognitive measures may be accounted for by several factors. Most notably, the coefficient of variation (the ratio of the standard deviation to the mean) was much larger for the ADAScog and MDRS than for the MMSE. This may reflect the greater 


\begin{tabular}{|c|c|c|c|c|c|}
\hline Test & $\begin{array}{l}\text { Mean scores on } \\
\text { treatment (SD) }\end{array}$ & $\begin{array}{l}\text { Mean scores } \\
\text { on placebo (SD) }\end{array}$ & $\begin{array}{l}\text { Treatment } \\
\text { effect (SE) }\end{array}$ & $p$ value & $\begin{array}{l}\text { Adjusted } \\
\text { p value* }\end{array}$ \\
\hline ADAScog & $22.5(6.9)$ & $24.4(9.4)$ & $-1.9(1.4)$ & 0.18 & 0.54 \\
\hline MMSE & 24.5 (3.2) & 22.5 (4.7) & $2.0(0.61)$ & 0.0044 & 0.018 \\
\hline MDRS & $108.3(17.13)$ & 108.5 (18.2) & $-0.2(1.9)$ & 0.98 & 0.98 \\
\hline MDRS attention & $31.0(5.1)$ & $31.1(5.2)$ & & & \\
\hline MDRS initiative & 25.9 (6.3) & $25.5(7.0)$ & & & \\
\hline CGI & $3.58(0.77)$ & $3.95(0.85)$ & -0.37 (NA) & 0.0056 & 0.022 \\
\hline
\end{tabular}

number of cognitive domains on the ADAScog and MDRS, or simply more familiarity among the investigators with administering the MMSE. There is no consensus on the most appropriate cognitive rating scale for $\mathrm{PD}$, which is why three different ones were used. The ADAScog was used as the primary outcome measure because it has been widely used in $\mathrm{AD}$ and has been shown to be responsive to cholinesterase inhibitors in $\mathrm{AD}$ and DLB.

While each cognitive scale measures several domains, there are differences in emphasis. Our analysis showed that the baseline scores on the ADAScog were moderately correlated with both the MMSE and MDRS (0.5-0.7). This suggests that the scales are measuring similar but not identical cognitive factors. One possible explanation then for why the ADAScog failed to pick up an effect of treatment is that it tends to emphasise the cognitive domains of memory and language, $^{2021}$ which may be more affected in AD than in PD. Improvements seen on the MMSE were not, however, attributable to any one subsection, such as those that focus on frontal executive function, nor were there clear benefits on the frontal executive subsection in the MDRS. Thus, we were not able to identify a specific cognitive domain that was preferentially improved by donepezil.

The MMSE was designed as a screening tool, and is thought to have limited sensitivity to small changes, which theoretically limits its usefulness in trials. ${ }^{27}$ However, in practice, it is commonly used in trials for $\mathrm{AD}, \mathrm{PD}$ and other disorders because of its familiarity and ease of use. ${ }^{27}$ It is somewhat surprising that it may have been more sensitive to changes in cognitive function in this study than other instruments that are specifically intended to measure change to due interventions.

Some authors believe that donepezil improves attention and behaviour rather than directly affecting memory. ${ }^{28}$ Change in behaviour and attention may be more readily apparent to the patient and family than specific cognitive changes. This may explain the finding of the clinical impression of change in the face of modest results on cognitive measures. One study in PD reported a selective improvement in a memory subscale of the MDRS, but this finding would not have been significant if correction had been made for multiple comparisons. ${ }^{19}$ Studies in $\mathrm{AD}$ have shown improvements in overt psychotic features and hallucinations with cholinesterase inhibitors. ${ }^{29}$ We did not observe any benefit on psychotic features of $\mathrm{PD}$, although the exclusion of severely psychotic patients may have limited the range of responses.

Overall, donepezil was well tolerated $(81 \%)$, and there was no evidence that it worsened PD motor symptoms. Consistent with previous trials, there was no evidence that donepezil worsened the motor features of $\mathrm{PD}$ as measured by the UPDRS total score or motor subscore.

The patients in this study and in two previous trials were diagnosed with PD and mild to moderate dementia. It is likely that this population included subjects who would meet pathological criteria for DLB. Current criteria differentiate these groups by certain clinical features. Unlike PD patients, DLB patients develop cognitive impairment at the time of or before motor manifestations. ${ }^{30}$ It is unclear if these subjects represent different disease entities, or subsets of the same disease that could be combined into one trial. Trials in DLB patients with the cholinesterase inhibitor rivastigmine show a similar magnitude of change on the MMSE (1.6 point difference between groups). ${ }^{31}$ Demented PD patients are a difficult population to recruit and retain in studies. The common pathophysiology and potential for similar responses to cholinergic agents suggests that combining demented PD patients and DLB patients in a stratified study may be a more efficient way to recruit for future trials.

There are two main methodological limitations to the interpretation of these study results. Firstly, this was a small study, and the three dropout patients reduced the study's initial power, inflating the potential for type 2 errors (false negative rate). Thus, the trend seen in this trial might indicate that treatment effects may really exist on the ADAScog or other measures that were not statistically significant in this study. This is a common problem with small clinical trials, and may be addressed by pooling these results with those of other published donepezil trials. As the analysis was paired, it is unlikely that dropouts introduced bias into the observed results, and the $14 \%$ dropout rate here is not greater than those seen in other studies. ${ }^{18} 32$

The second limitation is the potential for carryover or period effects to obscure the true treatment effects. This

\begin{tabular}{lcccl} 
Table 3 & Efficacy & results on BPRS and selected psychosis items & \\
\hline & $\begin{array}{l}\text { Scores on } \\
\text { treatment (SD) }\end{array}$ & $\begin{array}{l}\text { Scores on } \\
\text { placebo (SD) }\end{array}$ & $\begin{array}{l}\text { Treatment } \\
\text { effect (SE) }\end{array}$ & $\begin{array}{l}\text { Adjusted } \\
\text { p value* }\end{array}$ \\
\hline $\begin{array}{l}\text { BPRS total } \\
\text { Hostility }\end{array}$ & $32.9(9.6)$ & $33.2(7.9)$ & $-0.26(1.8)$ & $0.68(0.98)$ \\
$\begin{array}{l}\text { Suspiciousness } \\
\text { Hallucinatory }\end{array}$ & $1.5(0.8)$ & $1.4(0.2)$ & $0.1(0.2)$ & - \\
behaviour & $1.7(1.2)$ & $1.4(0.9)$ & $0.2(0.1)$ & - \\
$\begin{array}{l}\text { Unusual thought } \\
\text { content }\end{array}$ & $1.3(0.9)$ & $1.8(1.1)$ & $-0.1(0.3)$ & - \\
\hline
\end{tabular}

BPRS, Brief Psychosis Rating Scale. *Adjusted for multiple comparisons using Hommel method. 


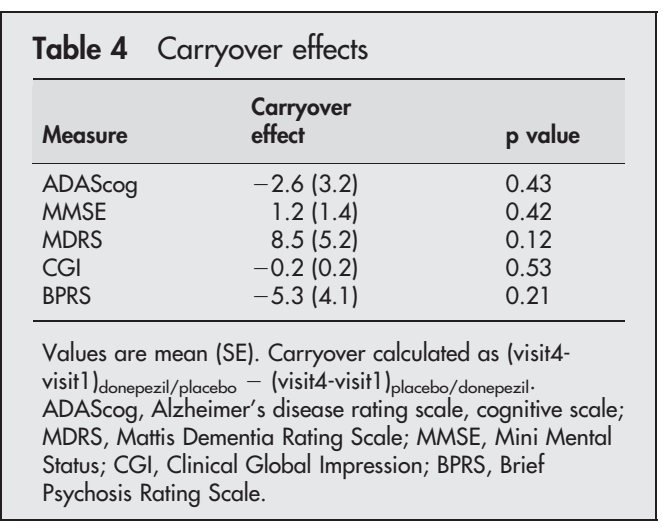

problem is inherent in crossover studies. In this study, persistent donepezil effects during the placebo period in the donepezil/placebo group would be expected to bias the results toward the null hypothesis and thus underestimate the true effect of donepezil. This concern is supported by the more marked differences observed between placebo and donepezil groups in the first period of the study (fig 2). While tests for carryover were not significant, this test may be underpowered because the carryover effect may be substantially smaller than the treatment effect for which the study was designed. Additionally, the carryover estimate was based on individual baseline measurements rather than means of two measurements as for the treatment effect. Thus, we cannot rule out the possibility that carryover effects on the ADAScog may still have played some role in reducing the observed favourable trend of donepezil treatment as measured by this scale. This problem was anticipated in the design of the trial, and a 6 week washout ( 17 half lives) was used for this drug, which has a 70 hour half life ${ }^{16}$ to ensure no pharmacokinetic or pharmacoydnamic carryover. Additionally, the efficacy analyses for the second period were based on visits 5 and 6 , which provided another 7 and 10 weeks for washout, respectively.

Period effects, defined as different results depending upon the period of the study, may also have affected the observed result. Regardless of the treatment sequence (donepezil/ placebo or placebo/donepezil), there was a trend toward better scores on the ADAScog with each of the visits following each baseline (fig 2). This may reflect placebo effects or, more likely, learning effects after repeat administrations of the test. Our secondary analysis, adjusting for both sequence and period effects in a repeated measures model, yields a nearly statistically significant result. The difference in statistical significance from the primary analysis reflects the reduced standard error from estimating period and sequence effects, accounting for some of the variability in the results. This suggests that these effects may have reduced the precision in the estimate of the benefits of donepezil. Parallel group studies eliminate concerns about carryover and period by treatment interactions, but would require a substantially larger sample size. ${ }^{33}$

This study examined the short term benefits of donepezil. The short term effects of cholinesterase inhibitors in $\mathrm{AD}$ are well established, but the long term effects and impact on independence remain controversial. A recent longer term clinical trial of donepezil in $\mathrm{AD}$ showed no benefit of donepezil on time to institutionalisation, death, or costs of care. $^{34}$ These results may not translate directly to $\mathrm{PD}$, because of differences in the progression and severity of dementia, and of differences in the relationship between cognitive and motor impairment. It will, however, be important to examine the impact of cholinesterase inhibitors in long term, practical trials of $\mathrm{PD}$
Table 5 Safety and tolerability measures during the time period of each exposure

\begin{tabular}{llll}
\hline & $\begin{array}{l}\text { Donepezil } \\
(\mathbf{n = 2 1 )}\end{array}$ & $\begin{array}{l}\text { Placebo } \\
(\mathbf{n}=\mathbf{2 0})\end{array}$ & p value \\
\hline $\begin{array}{l}\text { Tolerability } \\
\begin{array}{l}\text { Total UPDRS (mean } \\
\text { (SD)) }\end{array}\end{array}$ & $\begin{array}{l}17 / 21 \\
63.4(22.7)\end{array}$ & $\begin{array}{l}18 / 20 \\
65.7(21.3)\end{array}$ & 0.41 \\
$\begin{array}{llll}\text { Motor UPDRS (mean } \\
\text { (SD)) }\end{array}$ & $40.3(13.6)$ & $40.5(13.7)$ & 0.76 \\
Any AE & 11 & 9 & 0.64 \\
Nausea & 3 & 1 & 0.32 \\
Diarrhoea & 1 & 1 & 0.97 \\
Psychosis/agitation & 5 & 5 & 0.92 \\
Arrhythmia & 1 & 0 & 0.32 \\
\hline
\end{tabular}

UPDRS, Unified Parkinson's Disease Rating Scale (higher worse). AE, adverse event.

\section{CONCLUSIONS}

Although this is a small study, it is the largest reported randomised, blinded, placebo controlled clinical trial of a cholinesterase inhibitor in PD and dementia. Subjects were drawn from four centres, and the results are similar to those observed in a previous study, suggesting that these results may be generalisable. The results show that donepezil is well tolerated in this population, does not worsen PD, and that there is a place for donepezil in the treatment of PD dementia, although results are likely to be modest. Certain patient factors may predict greater responses to donepezil, but this study was not powered to detect such relationships. Dementia remains a largely untreated symptom in PD with severe consequences. Other agents in this class as well as in other classes, such as memantine, should be tested in PD dementia. Future studies should also address both milder and more severe forms of cognitive impairment in PD and could examine the long term effects on activities of daily living and independence.

\section{ACKNOWLEDGEMENTS}

This research was supported by the National Institutes of Neurological Disorders and Stroke, and by AG10124. A Siderowf is supported by grant K08-HS00004 from the Agency for Healthcare Research and Quality. T Simuni was supported by a grant from Northwestern Memorial Foundation. M Putt was supported by NIHCD P30-HD02679-14. KAI Inc. of Rockville, Maryland assisted in data collection and preparation.

\section{Authors' affiliations}

B Ravina, M Gillespie, A Crawley, National Institute of Neurological Disorders and Stroke, Bethesda, MD, USA

M Putt, J T Farrar, University of Pennsylvania, Center for Clinical Epidemiology and Biostatistics, Philadelphia, PA, USA

A Siderowf, S Reichwein, University of Pennsylvania, Department of Neurology, Philadelphia, PA, USA

H H Fernandez, University of Florida at Gainesville, Department of Neurology, Gainesville, FL, USA

M M Trieschmann, Brown University, Department of Neurology, Providence, RI, USA

T Simuni, Northwestern University, Department of Neurology, Chicago, IL, USA

Competing interests: none declared

\section{REFERENCES}

1 Van Den Eeden SK, Tanner CM, Bernstein AL, et al. Incidence of Parkinson's disease: variation by age, gender, and race/ethnicity. Am J Epidemiol 2003; 157:1015-22.

2 Litvan I, Bhatia KP, Burn DJ, et al. Movement Disorders Society Scientific Issues Committee report: SIC Task Force appraisal of clinical diagnostic criteria for Parkinsonian disorders. Mov Disord 2003;18:467-86.

3 Levy G, Schupf N, Tang MX, et al. Combined effect of age and severity on the risk of dementia in Parkinson's disease. Ann Neurol 2002;51:722-9. 
4 Hobson P, Meara J. Risk and incidence of dementia in a cohort of older subjects with Parkinson's disease in the United Kingdom. Mov Disord 2004; 19:1043-9

5 Levy G, Tang MX, Louis ED, et al. The association of incident dementia with mortality in PD. Neurology 2002;59:1708-13.

6 Louis ED, Marder K, Cote L, et al. Mortality from Parkinson disease. Arch Neurol 1997;54:260-4.

7 McKeith IG, Mosimann UP. Dementia with Lewy bodies and Parkinson's disease. Parkinsonism Relat Disord 2004;10(suppl 1):S15-18.

8 Hurtig HI, Trojanowski JQ, Galvin J et al. Alpha-synuclein cortical Lewy bodies correlate with dementia in Parkinson's disease. Neurology 2000;54:1916-21.

9 Perry EK, Curtis M, Dick DJ, et al. Cholinergic correlates of cognitive impairment in Parkinson's disease: comparisons with Alzheimer's disease. $J$ Neurol Neurosurg Psychiatry 1985;48:413-21.

10 Ruberg M, Ploska A, Javoy-Agid F, et al. Muscarinic binding and choline acetyltransferase activity in Parkinsonian subjects with reference to dementia. Brain Res 1982;232:129-39.

11 Shinotoh $\mathbf{H}$, Namba $\mathrm{H}$, Yamguchi $M$, et al. In vivo mapping of brain cholinergic function in Parkinson's disease and progressive supranuclear palsy. Adv Neurol 2001;86:249-55.

12 Candy JM, et al. Pathological changes in the nucleus of Meynert in Alzheimer's and Parkinson's diseases. J Neurol Sci 1983;59:277-89.

13 Rogers JD, Brogan D, Mirra SS. The nucleus basalis of Meynert in neurological disease: a quantitative morphological study. Ann Neurol 1985; 17:163-70.

14 Rogers SL, Friedhoff LT. The efficacy and safety of donepezil in patients with Alzheimer's disease: results of a US multicentre, randomized, double-blind, placebo-controlled trial. The Donepezil Study Group. Dementia 1996;7:293-303.

15 Rogers SL, Doody RS, Mohs RC, et al. Donepezil improves cognition and global function in Alzheimer disease: a 15-week, double-blind, placebocontrolled study. Donepezil Study Group. Arch Intern Med 1998:158:1021-31.

16 Wilkinson DG. The pharmacology of donepezil: a new treatment of Alzheimer's disease. Expert Opin Pharmacother 1999;1:121-35.

17 Richard IH, Justus AW, Greig NH, et al. Worsening of motor function and mood in a patient with Parkinson's disease after pharmacologic challenge with oral rivastigmine. Clin Neuropharmacol 2002;25:296-9.

18 Aarsland D, Laake K, Larsen JP, et al. Donepezil for cognitive impairment in Parkinson's disease: a randomised controlled study. J Neurol Neurosurg Psychiatry 2002;72:708-12.
19 Leroi I, Brandt J, Reich SG et al. Randomized placebo-controlled trial of donepezil in cognitive impairment in Parkinson's disease. Int J Geriatr Psychiatry 2004; 19:1-8.

20 Mohs RC, Cohen L. Alzheimer's Disease Assessment Scale (ADAS) Psychopharmacol Bull 1988:24:627-8.

21 Zec RF, Landreth ES, Vicari SK et al. Alzheimer disease assessment scale: useful for both early detection and staging of dementia of the Alzheimer type. Alzheimer Dis Assoc Disord 1992;6:89-102.

22 Paolo AM, Troster Al, Glatt SL, et al. Differentiation of the dementias of Alzheimer's and Parkinson's disease with the dementia rating scale. J Geriatr Psychiatry Neurol 1995;8:184-8.

23 Juncos JL, roberts VJ, Evatt ML, et al. Quetiapine improves psychotic symptoms and cognition in Parkinson's disease. Mov Disord 2004;19:29-35.

24 Siderowf A, McDermott M, Kieburtz K, et al. Test-retest reliability of the unified Parkinson's disease rating scale in patients with early Parkinson's disease: results from a multicenter clinical trial. Mov Disord 2002;17:758-63.

25 Senn S. Cross-over trials in clinical research. West Sussex: John Wiley \& Sons Ltd, 2002.

26 Hommel G. A stagewise rejective multiple test procedure based on a modified Bonferroni test. Biometrika 1988;75:383-386.

27 Rovner BW, Folstein MF. Mini-mental state exam in clinical practice. Hosp Pract (Off Ed) 1987;22:99, 103, 106, 110.

28 Cummings JL, Donohue JA, Brooks RL. The relationship between donepezil and behavioral disturbances in patients with Alzheimer's disease. Am J Geriatr Psychiatry 2000;8:134-40

29 Wynn ZJ, Cummings JL. Cholinesterase inhibitor therapies and neuropsychiatric manifestations of Alzheimer's disease. Dement Geriatr Cogn Disord 2004; 17:100-8.

30 McKeith IG, Perry EK, Perry RH. Report of the second dementia with Lewy body international workshop: diagnosis and treatment. Consortium on Dementia with Lewy Bodies. Neurology 1999;53:902-5.

31 McKeith I Del Ser T, Spano P, et al. Efficacy of rivastigmine in dementia with Lewy bodies: a randomised, double-blind, placebo-controlled international study. Lancet 2000;356:2031-6.

32 Rogers SL, Farlow MR, Doody RS, et al. A 24-week, double-blind, placebocontrolled trial of donepezil in patients with Alzheimer's disease. Donepezil Study Group. Neurology 1998;50:136-45.

33 Putt ME, Ravina B. Randomized, placebo-controlled, parallel group versus crossover study designs for the study of dementia in Parkinson's disease. Control Clin Trials 2002;23:111-26.

34 Group AC. Long-term donepezil treatment in 565 patients with Alzheimer's disease (AD2000): randomised double-blind trial. The Lancet 2004:363:2105-15. 